\title{
Kernos
}

Revue internationale et pluridisciplinaire de religion grecque antique

$23 \mid 2010$

Varia

\section{Anders Holm RASMUSSEN, Susanne William RASMUSSEN (éds), Religion and Society. Rituals, Resources and Identity in the Ancient Graeco- Roman World. The BOMOS-Conferences 2002-2005}

\section{Stéphanie Paul}

\section{OpenEdition}

Journals

Édition électronique

URL : http://journals.openedition.org/kernos/1642

DOI : 10.4000/kernos.1642

ISSN : 2034-7871

Éditeur

Centre international d'étude de la religion grecque antique

Édition imprimée

Date de publication : 1 janvier 2010

Pagination : 391-394

ISSN : 0776-3824

Référence électronique

Stéphanie Paul, «Anders Holm rasmussen, Susanne William rasmussen (éds), Religion and Society. Rituals, Resources and Identity in the Ancient Graeco-Roman World. The BOMOS-Conferences 2002-2005 », Kernos [En ligne], 23 | 2010, mis en ligne le 15 septembre 2011, consulté le 21 septembre 2020. URL : http://journals.openedition.org/kernos/1642 ; DOI : https://doi.org/10.4000/kernos.1642

Ce document a été généré automatiquement le 21 septembre 2020

Kernos 


\title{
Anders Holm RASMUSSEN, Susanne William RASMUSSEN (éds), Religion and Society. Rituals, Resources and Identity in the Ancient Graeco- Roman World. The BOMOS- Conferences 2002-2005
}

\author{
Stéphanie Paul
}

\section{RÉFÉRENCE}

Anders Holm RASMUSSEN, Susanne William RASMUSSEN (éds), Religion and Society. Rituals, Resources and Identity in the Ancient Graeco-Roman World. The BOMOS-Conferences 2002-2005, Roma, Edizioni Quasar, 2008. 1 vol. $21 \times 29,5$ cm, 296 p. (Analecta Romana Instituti Danici, suppl. 40). ISBN : 978-88-7140-371-7.

1 Ce volume reprend les actes de trois conférences organisées par le groupe de recherche BOMOS et tenues à l'University of Southern Denmark en 2002, à l'Aarhus University en 2003, et à l'Institut danois de Rome en 2005. La thématique commune de ces activités porte sur les interactions entre religion et société dans le monde gréco-romain et s'articule selon trois axes, qui constituaient chacun le propos d'une des conférences et qui font l'objet d'un chapitre du livre : 1) Ritual and Cult; 2) Resources and Manpower; 3) Religion and Identity. Pas moins de vingt-huit articles y étant rassemblés, il eût été difficile de rendre compte de chacun d'eux en détail en ce lieu. J'ai donc privilégié l'un ou l'autre point qui a retenu mon attention.

2 Au-delà de la division en trois parties qui scandent ce volume, on constate que plusieurs thèmes reviennent de manière transversale. Par exemple, le sujet du 
personnel de culte, plus spécifiquement des prêtrises, est récurrent. Dans la première partie, A.H. Rasmussen (p. 71-80) veut mettre en évidence le rôle fondamental du prêtre grec au sein du rituel, arguant que celui-ci était, sauf exception, indispensable à son bon fonctionnement. R. prend là le contrepied d'une série d'opinions modernes qui ont eu tendance à réduire le prêtre grec à un simple magistrat, opinions qui ont toutefois été fortement nuancées dans de récentes études ${ }^{1}$. Dans la deuxième partie, le même A. se penche sur les aspects économiques des prêtrises (p. 133-137), sur lesquels les contrats de ventes, provenant d'Asie mineure et des îles de l'Égée, nous livrent de précieuses informations. Les sommes importantes qui étaient parfois déboursées pour leur acquisition étaient compensées par une série d'avantages financiers, qui comprenaient l'exemption de certaines liturgies ou l'obtention des parts d'honneur des sacrifices. D'après l'exemple de la vente de la prêtrise d'Hermès Enagonios à Cos, R. émet en outre l'hypothèse que le prêtre devait assumer les frais d'achat des victimes sacrificielles. Cette hypothèse est sujette à discussion, car il apparaît assez clairement dans ce document et d'autres du même type que le coût des sacrifices était pris en charge soit par la cité, via les trésoriers, ou plus rarement par le dieu, soit par les sacrifiants eux-mêmes ${ }^{2}$. C'est encore des ventes de prêtrise qu'il est question dans un article de K. Buraselis (p. 125-131), où sont examinés à leur tour les aspects idéologiques de la mise en place d'une telle procédure vis-à-vis de la cité, ainsi que l'avantage financier qu'elle pouvait représenter pour elle.

3 Les lieux de culte constituent une autre thématique abordée de manière transversale tout au long de l'ouvrage. B. Forsén s'interroge, en introduction de la deuxième partie, sur les moyens à mettre en œuvre pour construire un temple grec, en prenant pour exemple le temple archaïque d'Agios Elias en Arcadie (p. 119-124). Chr. Kvium se penche, quant à lui, sur les distinctions entre propriété privée, publique ou sacrée à la fin de la période républicaine et au début de l'Empire romain (p. 160-164). Enfin, la topographie sacrée de deux lieux spécifiques est envisagée, en rapport avec la question de l'identité, dans deux contributions différentes. J. Whitley s'intéresse aux sanctuaires de Praisos en Crète (p. 235-248), et B. Forsén à ceux du sud-est de l'Arcadie (p. 248-258).

4 En guise d'introduction à la première partie, sur le thème "ritual and cult ", J.P. Sørensen propose quelques réflexions théoriques (p. 13-22). Trois contributions sont ensuite centrées sur des pratiques rituelles transmises par les auteurs littéraires. Du côté romain, J.A. North analyse le compte rendu par Tite-Live de la dédicace du temple du Capitole (p. 23-36) et J.F. Miller passe en revue les mentions du temple d'Apollon sur le Palatin chez les poètes augustéens (p. 43-53). Du côté grec, L. Bruit Zaidman se penche d'une manière plus générale sur l'interaction entre mythe et rituel dans la Périégèse de Pausanias (p. 81-89). La communication avec le divin, qui est l'objectif premier de la pratique rituelle, fait l'objet de deux autres contributions de cette première partie. S.W. Rasmussen consacre une étude à l'expiation des prodiges, indispensable à l'équilibre de la société romaine (p. 37-42). V. Rosenberger envisage la pratique de la consultation oraculaire en Grèce sous deux aspects différents: la consécration d'offrandes gratulatoires dans les sanctuaires oraculaires d'une part, et la fréquentation de ces sanctuaires d'autre part (p. 91-106). R. constate que ces offrandes, en plus de leur fonction première - remercier le dieu -, constituent également un moyen d'auto-représentation, puisque, si l'on suit la théorie de Marcel Mauss sur le don, le lien avec le donneur n'en est jamais complètement effacé. Son parcours de la fréquentation des oracles ne s'avère pas totalement concluant, car il se heurte à l'hétérogénéité des sources et de leur répartition, un problème méthodologique 
pourtant souligné par l'A. elle-même. Enfin, cette première partie comporte encore une étude de I. Mæhle (p. 61-69) sur le rôle des cultes féminins comme moyen d'influence lors de la lutte des classes de la République romaine, et deux autres contributions se fondant sur des sources épigraphiques. I. Gradel (p. 55-60) tente de dégager les usages qui présidaient aux titulatures des empereurs romains dans leurs épitaphes. K.R. Kristensen interroge les interactions entre religion et loi en Crète, à travers la figure d'Apollon, dont le rôle dans la création et la préservation des lois écrites transparaît à la fois dans le fait que ces lois étaient affichées dans son temple, et dans ses épiclèses, qui sont en étroite relation avec ses cultes de Délos et de Delphes (p. 107-115).

5 La deuxième partie se concentre plus étroitement sur les ressources matérielles et les effectifs humains nécessaires à l'organisation du religieux. Outre les articles qu'on a déjà mentionnés sur les prêtres, la construction des temples et la propriété, elle comprend deux articles autour de la déesse Aphrodite et ses œuvres. Dans une étude sur les dédicaces des magistrats à Aphrodite (p. 139-150), J. Wallensten remet en question l'interprétation selon laquelle ces dédicaces doivent être expliquées par les compétences de la déesse en matière de concorde et d'harmonie. W. y voit la marque de l'individualisation de la religion et la recherche d'une relation plus personnelle avec la divinité, caractéristiques selon elle de l'époque hellénistique. S.W. Rasmussen reprend une partie du dossier problématique de la prostitution sacrée, à savoir le culte de Vénus sur le mont Éryx et celui de Vénus Érycina à Rome (p. 151-157).

6 C'est le thème de l'identité qui est au centre de la troisième et dernière partie de l'ouvrage. La mémoire, qui en est un élément constitutif, est le sujet de la contribution de S. Price (p. 167-178) et sa transmission, celle de V. Rosenberger (p. 225-233), consacrée au marbre de Paros. Le thème de l'héritage du passé est l'objet de l'étude de L. Bruit Zaidman, sur la notion d'archaïsme en Grèce, d'Hérodote à Pausanias (p. 179-188). Cette troisième partie comporte encore deux études sur l'intégration de nouveaux cultes. J.A. Krasilnikoff se penche sur le contexte de l'intégration du culte de Pan en Attique lors des guerres médiques (p. 189-200) et K. Buraselis, sur celui des souverains dans la religion "traditionnelle " à l'époque hellénistique (p. 215-223). R. Parker s'intéresse au sujet des cultes des subdivisions sociales en Grèce à travers les figures des theoi patrôoi, particulièrement dans les cités de Thasos, de Cos et d'Éphèse, et, plus largement, pose la question de la définition des groupes appelés "phratries » (p. 201-214). L'étude est suivie d'une annexe très utile des attestions de ces cultes. Du côté romain, Chr. Kvium se place dans la même lignée en proposant quelques réflexions sur les gentes et gentiles archaïques (p. 267-285). Enfin, l'ouvrage se termine par une étude de J.M. Rosenløv (p. 287-294) sur les théories cosmologiques des philosophes latins et par une note de J.E. Skydsgaard (p.295-296) sur le cheval de César.

7 À la fin de ce parcours, indépendamment de la qualité individuelle des articles, on pourra regretter le manque d'homogénéité de cet ouvrage. En dépit de l'agencement en trois chapitres thématiques, le volume ressemble à une juxtaposition de contributions sans fil conducteur apparent, et l'absence d'une introduction développée ou de conclusions générales de la part des éditeurs est, de ce point de vue, assez révélatrice. À vrai dire, l'éclatement des sujets abordés semble inévitable quand on voit l'ampleur des thèmes proposés, en particulier ceux des première et dernière parties. Une autre réserve que l'on pourra émettre est la longue période qui sépare la tenue des conférences de la parution des actes. Par conséquent, certains des articles sont déjà dépassés, notamment d'un point de vue bibliographique, les publications récentes 
n'ayant pas pu être prises en compte. Ces réserves mises à part, ce volume propose quelques contributions qui seront bien utiles aux spécialistes des questions religieuses.

\section{NOTES}

1. V. Pirenne-Delforge - St. Georgoudi, "Personnel de culte : monde grec ", ThesCRA V (2005), p. 2-65; B. DIGNAS - K. TRAMPEDACH (éds), Practitioners of the Divine. Greek Priests and Religious Officials from Homer to Heliodorus, Washington/Cambridge, 2008.

2. ICos ED 145, 1. 50. Sur ces aspects, voir H.-U. WIEMER, « Käufliche Priestertümer im hellenistischen Kos », Chiron 33 (2003), p. 294.

\section{AUTEURS}

STÉPHANIE PAUL

Université de Liège 